\title{
Exploration of variance, autocorrelation, and skewness of deviations from lactation curves as resilience indicators for breeding
}

\author{
M. Poppe, ${ }^{1 *}$ ( $)$ R. F. Veerkamp, ${ }^{1} \odot$ M. L. van Pelt, ${ }^{2} \odot$ and H. A. Mulder ${ }^{1} \odot$ \\ ${ }^{1}$ Wageningen University and Research, Animal Breeding and Genomics, PO Box 338, $6700 \mathrm{AH}$ Wageningen, the Netherlands \\ ${ }^{2}$ Cooperation CRV, Animal Evaluation Unit, PO Box 454, 6800 AL Arnhem, the Netherlands
}

\begin{abstract}
The ability of a cow to cope with environmental disturbances, such as pathogens or heat waves, is called resilience. To improve resilience through breeding, we need resilience indicators, which could be based on the fluctuation patterns in milk yield resulting from disturbances. The aim of this study was to explore 3 traits that describe fluctuations in milk yield as indicators for breeding resilient cows: the variance, autocorrelation, and skewness of the deviations from individual lactation curves. We used daily milk yield records of 198,754 first-parity cows, recorded by automatic milking systems. First, we estimated a lactation curve for each cow using 4 different methods: moving average, moving median, quantile regression, and Wilmink curve. We then calculated the log-transformed variance (LnVar), lag-1 autocorrelation $\left(\mathrm{r}_{\text {auto }}\right)$, and skewness (Skew) of the daily deviations from these curves as resilience indicators. A genetic analysis of the resilience indicators was performed, and genetic correlations between resilience indicators and health, longevity, fertility, metabolic, and production traits were estimated. The heritabilities differed between LnVar (0.20 to 0.24), $\mathrm{r}_{\text {auto }}$ (0.08 to 0.10 ), and Skew (0.01 to 0.02), and the genetic correlations among the indicators were weak to moderate. For $\mathrm{r}_{\text {auto }}$ and Skew, genetic correlations with health, longevity, fertility, and metabolic traits were weak or the opposite of what we expected. Therefore, $r_{\text {auto }}$ and Skew have limited value as resilience indicators. However, lower LnVar was genetically associated with better udder health (genetic correlations from -0.22 to $-0.32)$, better longevity $(-0.28$ to -0.34$)$, less ketosis $(-0.27$ to -0.33$)$, better fertility $(-0.06$ to -0.17$)$, higher BCS $(-0.29$ to -0.40$)$, and greater dry matter intake $(-0.53$ to -0.66$)$ at the same level of milk yield.
\end{abstract}

Received July 18, 2019.

Accepted October 3, 2019.

*Corresponding author: marieke.poppe@wur.nl
These correlations support LnVar as an indicator of resilience. Of all 4 curve-fitting methods, LnVar based on quantile regression systematically had the strongest genetic correlations with health, longevity, and fertility traits. Thus, quantile regression is considered the best curve-fitting method. In conclusion, LnVar based on deviations from a quantile regression curve is a promising resilience indicator that can be used to breed cows that are better at coping with disturbances.

Key words: resilience, variance, milk yield, automatic milking system, dairy cow

\section{INTRODUCTION}

Cows differ in their ability to cope with environmental disturbances such as pathogens, heat waves, and changes in feed composition and feed quantity. A cow that is unaffected by a disturbance, or that quickly returns to her normal level of functioning, is labeled resilient (Colditz and Hine, 2016; Berghof et al., 2019b). Resilience could be improved through genetic selection, but to do so we need to measure it in individual cows. Several studies have quantified responses to and recovery from an experimental disturbance in animals (Friggens et al., 2016; Revilla et al., 2019). However, such challenge experiments cannot be carried out routinely on commercial farms. Moreover, challenge experiments focus on a single type of disturbance, whereas our interest is in improving the general resilience to unknown disturbances.

Alternatively, a data-driven approach can be used. Scheffer et al. (2018) proposed methods to quantify resilience in cows using frequently measured data. These methods rely on the assumption that cows are constantly subject to unknown disturbances, which result in fluctuations in frequently measured traits. Cows with few fluctuations are less affected by disturbances than are cows with more fluctuations. Therefore, the fluctuation pattern is expected to be informative about resilience. Several resilience indicators describing fluctuations in frequently measured traits have been suggested, such as the variance of the trait, which indicates the variability 
of the frequently measured trait, the lag-1 autocorrelation of the trait, which indicates stretches of values above or below the expected level, and skewness of the trait, which indicates asymmetry (Scheffer et al., 2018; Berghof et al., 2019b). Until now, it has been difficult to validate resilience indicators by relating them to response to disturbances, because of the lack of data on disturbances. However, genetic analysis can be used to better understand the biology of a trait and, as such, increase our understanding of new resilience indicators, similar to validating subjectively scored traits such as BCS (Veerkamp et al., 2002).

A trait that is frequently measured and that shows response to disturbances is milk yield. Variance of milk yield has been studied by several researchers. Elgersma et al. (2018) showed that variance of daily recorded milk yield was heritable and that cows with a lower variance had genetically better udder health, less ketosis, and better longevity. Therefore, variance of daily milk yield has potential as a resilience indicator. However, Elgersma et al. (2018) did not account for the lactation curve shape, which is expected to influence the level of variance in milk yield. Other studies have shown genetic variation in environmental variance in milk yield using a random regression model, which did account for lactation curve shape (Rönnegård et al., 2013; Vandenplas et al., 2013; Ehsaninia et al., 2019). However, these authors used test-day records, which are not frequent enough to detect all fluctuations in milk yield. Overall, there is room for improvement of variance in milk yield as a resilience indicator. Furthermore, autocorrelation and skewness of daily recorded milk yield may provide additional information about resilience.

The aim of this study was to explore the use of variance, autocorrelation, and skewness of deviations in daily milk yield level from a lactation curve as indicators for breeding resilient cows. We explored 4 methods to fit the lactation curve: moving average, moving median, quantile regression, and Wilmink curve. The heritabilities of all resilience indicators were estimated, as well as genetic correlations among the resilience indicators and genetic correlations among the curve-fitting methods. In addition, genetic correlations between the resilience indicators and health, production, longevity, fertility, and metabolic traits were estimated.

\section{MATERIALS AND METHODS}

In this study, we performed a genetic analysis on 3 potential resilience indicators. The initial data contained $1,782,373,113$ milk yield records from $1,120,550$ cows, obtained during single milk visits to automatic milking systems (AMS) and conventional milking systems. Po- tential resilience indicators were calculated from these data for 198,754 first-parity Holstein Friesian cows milked by AMS (see Data Editing section). Calculation of potential resilience indicators was performed in 2 steps: (1) fitting of individual lactation curves and (2) defining resilience indicators based on deviations from lactation curves. We will first describe the 2 steps that generated the resilience indicators, followed by data editing and genetic analysis.

\section{Fitting Individual Lactation Curves}

The aim of fitting individual lactation curves for each cow based on daily milk yield records was to predict the expected milk yield of a cow at each day. Ideally, a fitted lactation curve would be as close as possible to the curve that a cow would have realized in the absence of disturbances, because then deviations from the curve would contain the most information about responses to disturbances. The deviations from such a curve could thus be used to indicate resilience (see Figure 1 for an example of a fitted lactation curve and the deviations from that lactation curve). Fitting a lactation curve that a cow would have realized in the absence of disturbances was difficult, because information about disturbances was lacking, and disturbances may even be cow-specific and unknown. Therefore, different methods were explored for fitting the individual lactation curves using only the daily milk yield records as input. The choice of the best curve-fitting method is, by definition, arbitrary. However, the results of this study will generate insight into which method has most potential and how sensitive genetic parameters are when changing the curve-fitting method. There are 2 generic ways of fitting a trend through a time series: nonparametric trend estimation and model-based estimation (Brockwell and Davis, 2016). Because we did not know which method would generate the best results, we decided to explore both. We used 4 methods: 2 nonparametric (moving average and moving median) and 2 model-based methods [the Wilmink lactation curve (Wilmink, 1987) and a quantile polynomial regression method]. See Figure 2 for illustrations of the 4 lactation curve-fitting methods.

Nonparametric Trend Estimation. The first nonparametric method was a 2 -sided moving average filter with a window of $21 \mathrm{~d}$. This means that the expected milk yield on a certain day is the average of the milk yield of the $10 \mathrm{~d}$ before that day, the $10 \mathrm{~d}$ after that day, and the day itself. Window sizes different from 21 $\mathrm{d}$ were explored as well. However, based on visual inspection of fitted trends for random cows from the data set, we decided to focus on a window of $21 \mathrm{~d}$. Because a moving average is relatively sensitive to drops in milk yield, we also decided to fit a moving median filter 
with a window of $21 \mathrm{~d}$. A moving median is the same as a moving average, but the expected milk yield is the median, and not the mean, of a series of milk yield records. Both the moving average and the moving median filter were applied using the rollapply function in the zoo package in $\mathrm{R}$ (Zeileis and Grothendieck, 2005). The advantage of the moving average and moving median is that they are flexible, because expected yields are dependent only on the data points that are close in time. However, the risk is that the moving average and moving median may be too flexible, which results in lost information on drops in milk yield in the deviations of the curve.

Model Building. The first model-based method was the Wilmink lactation curve (Wilmink, 1987):

$$
\text { yield }_{t}=\beta_{0}+\beta_{1} t+\beta_{2} e^{-0.05 t}+\varepsilon,
$$

where yield $_{t}$ is the observed milk yield on DIM $t, \beta_{0}$ is related to the level of production, $\beta_{1}$ describes the production decrease after the peak yield, $\beta_{2}$ describes the increase in milk yield at the start of the lactation, and $\varepsilon$ is the error term. The regression coefficients were estimated for each cow using the $\mathrm{lm}$ function in $\mathrm{R}$ and were then used to calculate the expected lactation curves. The advantage of the Wilmink curve is that it is a commonly used regression method for fitting lactation curves (Schaeffer et al., 2000; CRV, 2018a) and is therefore easy to understand. However, it might not fit the data for individual cows well enough, resulting in incomplete removal of the general trend.


Figure 1. (A) Observed (black line) and predicted (blue line) daily milk yield as a function of time after calving (in days) of an example cow. (B) Deviations from predicted milk yield as a function of time after calving (in days) of the same example cow. 



Figure 2. Examples of lactation curve-fitting methods for an example cow. Black lines show observed milk yield, and blue lines show predicted milk yield; (A) moving average, (B) moving median, (C) Wilmink curve, (D) quantile regression. 
The second model-based method was fourth-order polynomial quantile regression using a 0.7 quantile:

$$
\text { yield }_{t}=\beta_{0}+\beta_{1} t+\beta_{2} t^{2}+\beta_{3} t^{3}+\beta_{4} t^{4}+\varepsilon,
$$

where yield $d_{t}$ is the observed milk yield on DIM $t$ and $\varepsilon$ is the error term. Polynomial regression is commonly used in time series analysis (Brockwell and Davis, 2016). Similarly, most test-day models used in genetic evaluation use Legendre polynomials with random regression (van der Werf et al., 1998; Pool and Meuwissen, 2000; Swalve, 2000; CRV, 2018a). The advantage of fourthorder polynomial regression is that it is more flexible than a Wilmink curve, partly because of additional parameters, but the risk of being too flexible is smaller than for the trend-estimation methods. Quantile regression was used instead of classical linear regression, to make the resulting curves less sensitive to drops in milk yield and thus closer to the potential curves in the absence of disturbances. Whereas classical regression models estimate the conditional mean milk yield given certain values of DIM, quantile regression models estimate the conditional median or other quantile (Koenker, 2005). By using a quantile higher than 0.5, low milk yield values have less influence on the predicted milk yield curve than do high milk yield values. In other words, drops in milk yield have less influence on the predicted milk yield curve when using quantile regression with a quantile greater than 0.5 than when using classical linear regression, and the negative deviations from the quantile regression curve are larger. As a result, a quantile regression curve using a quantile greater than 0.5 was expected to better match the potential milk yield in absence of disturbances than was a classical linear regression curve and, thus, was expected to generate deviations that contain more information on resilience. Different quantiles higher than 0.5 were explored, but upon visual inspection of fitted curves for random cows from the data set, we decided to focus only on the 0.7 quantile. The regression coefficients of the fourth-order polynomial quantile regression model using the 0.7 quantile were estimated for each cow, using the quantreg package (Koenker, 2018) and the poly function in R. The estimated regression coefficients for each cow were then used to calculate individual expected lactation curves.

\section{Defining Resilience Indicators Based on Deviations from Lactation Curves}

The deviations from the fitted lactation curves $($ yield- $\widehat{\text { yield }})$ were expected to contain information about responses to environmental disturbances, and they were therefore used to calculate 3 potential resilience indicators: the variance, the lag- 1 autocorrelation, and the skewness of the deviations. Low variance of the deviations was expected to indicate good resilience, because resilient cows have a smaller range of deviations from their lactation curve than do less-resilient cows. A low lag-1 autocorrelation of the deviations was expected to indicate good resilience, because resilient cows have fewer and shorter stretches of negative deviations than do less-resilient cows. A skewness of the deviations close to zero was expected to indicate good resilience, because resilient cows have as many positive as negative deviations, whereas less-resilient cows have more negative than positive deviations (Scheffer, 2009; Scheffer et al., 2018; Berghof et al., 2019b). Because the lactation curve models had poor fit at the beginning and end of lactation, and because the moving average and moving median were based on less than $21 \mathrm{~d}$ in the beginning and end of lactation, the first and last 10 DIM of each cow were excluded from calculation of the resilience indicators.

The variance of the deviations was transformed with a natural logarithm, which made the trait normally distributed upon visual inspection. The transformation made the resulting genetic variance directly comparable to previous work on heritable variance in residual variance, which used an exponential model of the variance (SanCristobal-Gaudy et al., 1998; Hill and Mulder, 2010; Sell-Kubiak et al., 2015). From this point forward in this paper, log-transformed variance of deviations from a lactation curve will be referred to as LnVar, lag-1 autocorrelation will be referred to as $\mathbf{r}_{\text {auto }}$, and skewness will be referred to as Skew. Because each resilience indicator was calculated for 4 curve-fitting methods, each cow had in total 12 potential resilience indicators. In addition to LnVar, $\mathrm{r}_{\text {auto }}$, and Skew, the average daily milk yield and the natural log of the variance of the raw daily milk yield records (RawVar) were calculated for each cow. RawVar was used to compare our results to those of Elgersma et al. (2018), and the average daily milk yield was used to adjust genetic correlations between the resilience indicators and the health, longevity, fertility, and metabolic traits for milk yield level (see Genetic Analysis section).

\section{Data Editing}

The initial data set was provided by Cooperation CRV and CRV BV (Arnhem, the Netherlands), and contained milk yield records obtained during single milk visits to AMS and conventional milking systems, resulting in multiple records per day for each cow. The data consisted of 1,782,373,113 milk yield records from 1,120,550 cows, recorded between 1998 and 2018. How- 
ever, the resilience indicators were calculated only for first-parity Holstein Friesian cows that were milked by AMS, that were herd-book registered, that calved after $640 \mathrm{~d}$ of age and before Jun. 1, 2017, and that had no more than $5 \%$ missing daily milk yield records. Moreover, the resilience indicators were only based on the first 350 DIM. Daily milk yield records were obtained from the records of single AMS visits by summing the milk yield of the single AMS visits per day for each cow. However, the milk yield of the first AMS visit of each day was partly assigned to the previous day, depending on the milk produced per minute since the previous AMS visit and the time between the previous AMS visit and midnight. After we had calculated the resilience indicators, resilience indicator records of individual cows were set to missing if they deviated more than 4 standard deviations (SD) from the mean of that resilience indicator. To adjust for herd, year of calving, and season of calving, herd-year-season classes were made with 4 seasons: January to March, April to June, July to September, and October to December. Herd-year-season classes with less than 5 cows were removed. Finally, 198,754 cows were used for the genetic analysis of the resilience indicators. Data editing was performed using the AWK programming language (Aho et al., 1988) and R (version 3.2.2; R Project for Statistical Computing, Vienna, Austria). All data editing steps, with information on the number of remaining records and number of cows after each editing step, are shown in Table 1.

\section{Genetic Analysis}

For all resilience indicators and average daily milk yield, heritabilities and EBV were estimated with univariate analyses, and genetic correlations among traits were estimated with bivariate analyses using ASReml 4.1 (Gilmour et al., 2015). The pedigree included 5 generations of ancestors.

Univariate Analysis. Heritabilities and EBV were estimated using the following univariate linear mixed animal model:

$$
\mathbf{y}=\mathbf{X b}+\mathbf{Z a}+\mathbf{e},
$$

where $\mathbf{y}$ was a vector with observations of a certain resilience indicator; $\mathbf{b}$ was a vector containing fixed effects, which were herd-year-season, age at first calving in months, and lactation length (remaining number of days after removing the first and last 10 DIM) in 7 classes, each containing a range of $40 \mathrm{~d}$ (50 to $90 \mathrm{~d}, 91$ to $130 \mathrm{~d}$, and so on); a was a vector containing the additive genetic effects, $\mathbf{a} \sim N\left(0, \mathbf{A} \sigma_{a}^{2}\right)$, where $\mathbf{A}$ is the additive genetic relationship matrix and $\sigma_{a}^{2}$ is the additive genetic variance; and $\mathbf{e}$ was a vector containing the

Table 1. Data editing steps and numbers of records, missing records, and cows present after each editing step

\begin{tabular}{|c|c|c|c|}
\hline Editing step & $\begin{array}{l}\text { Number } \\
\text { of records }\end{array}$ & $\begin{array}{l}\text { Number of } \\
\text { missing records }\end{array}$ & $\begin{array}{l}\text { Number } \\
\text { of cows }\end{array}$ \\
\hline Original data set & $1,782,373,113$ & 0 & $1,120,550$ \\
\hline Select cows with parity 1 & $537,289,288$ & 0 & 774,241 \\
\hline Select cows milked by automatic milking system & $450,627,626$ & 0 & 588,541 \\
\hline Select cows that are registered and at least $87.5 \%$ Holstein Friesian & $354,900,725$ & 0 & 457,607 \\
\hline Remove records with 0 milk yield & $354,899,345$ & 0 & 457,607 \\
\hline Remove cows that moved between herds & $351,722,320$ & 0 & 453,535 \\
\hline Remove duplicate records & $345,144,971$ & 0 & 453,535 \\
\hline Calculate daily milk yield & $128,155,982$ & $14,171,909$ & 453,251 \\
\hline Remove records at more than 350 DIM & $116,450,351$ & $13,038,628$ & 453,251 \\
\hline Remove cows with fewer than 20 records $^{1}$ & $116,388,475$ & $12,670,122$ & 446,158 \\
\hline Remove cows that calved before $640 \mathrm{~d}$ of age & $115,913,747$ & $12,608,713$ & 444,281 \\
\hline Set outliers to missing based on Wilmink curve (CRV, 2018a) & $115,747,841$ & $12,774,619$ & 444,281 \\
\hline Set milk yield greater than $100 \mathrm{~kg}$ to missing & $115,747,816$ & $12,774,644$ & 444,281 \\
\hline Remove cows that have their first milk yield record after 14 DIM & $108,031,488$ & $8,005,087$ & 402,054 \\
\hline Fit lactation curves and remove cows with fewer than 50 records & $100,171,599$ & $7,002,276$ & 378,480 \\
\hline Aggregate daily milk yield records to resilience indicators & 378,480 & 0 & 378,480 \\
\hline $\begin{array}{l}\text { Remove cows with an average milk yield less than the mean herd } \\
\text { yield } \pm 4 \mathrm{SD}\end{array}$ & 378,364 & 0 & 378,364 \\
\hline Remove cows that calved after Jun. 1, 2017 & 334,387 & 0 & 334,387 \\
\hline $\begin{array}{l}\text { Remove cows for which more than } 5 \% \text { of their entire lactation } \\
\text { consisted of missing records }\end{array}$ & 255,096 & 0 & 255,096 \\
\hline $\begin{array}{l}\text { Set resilience indicator records to missing if they exceed mean } \pm 4 \\
\text { SD and remove cows with a missing resilience indicator based } \\
\text { on all curve-fitting methods }\end{array}$ & 254,788 & 0 & 254,788 \\
\hline Remove herd $\times$ year $\times$ season classes with fewer than 5 cows & 198,754 & 0 & 198,754 \\
\hline
\end{tabular}

${ }^{1}$ To fit a Wilmink curve, a sufficient number of records was needed. 
residuals $\mathbf{e} \sim N\left(0, \mathbf{I} \sigma_{e}^{2}\right)$, where $\mathbf{I}$ is the identity matrix and $\sigma_{e}^{2}$ is the residual variance; $\mathbf{X}$ and $\mathbf{Z}$ were incidence matrices linking the records in $\mathbf{y}$ to the fixed effects and additive genetic effects, respectively. For the LnVar traits and RawVar, a genetic coefficient of variation (GCV) was calculated as $\sigma_{a}$, because using the lntransformation of the variance assumes an exponential model, and GCV in the exponential model for variance is equal to $\sigma_{a}$ (see Mulder et al., 2007). For the other resilience indicators, a GCV was calculated as $\frac{\sigma_{a}}{\mu}$, where $\mu$ was the mean of the resilience indicator.

Bivariate Analysis. Genetic correlations between the different resilience indicators, between the same resilience indicators based on different lactation curvefitting methods, and between the resilience indicators and average daily milk yield, were estimated using the following bivariate mixed animal model:

$$
\left[\begin{array}{l}
\mathbf{y}_{1} \\
\mathbf{y}_{2}
\end{array}\right]=\left[\begin{array}{cc}
\mathbf{X}_{1} & 0 \\
0 & \mathbf{X}_{2}
\end{array}\right]\left[\begin{array}{l}
\mathbf{b}_{1} \\
\mathbf{b}_{2}
\end{array}\right]+\left[\begin{array}{cc}
\mathbf{Z}_{1} & 0 \\
0 & \mathbf{Z}_{2}
\end{array}\right]\left[\begin{array}{l}
\mathbf{a}_{1} \\
\mathbf{a}_{2}
\end{array}\right]+\left[\begin{array}{l}
\mathbf{e}_{1} \\
\mathbf{e}_{2}
\end{array}\right]
$$

where $\mathbf{y}_{i}$ was a vector with observations on trait $i$; $\mathbf{b}_{i}$ was a vector with the fixed effects for trait $i$, which were the same as in the univariate analysis; $\mathbf{a}_{i}$ was a vector with the additive genetic effects for trait $i$; and $\mathbf{e}_{i}$ was a vector with the residuals for trait $i ; \mathbf{X}_{i}$ and $\mathbf{Z}_{i}$ were incidence matrices linking the records in $\mathbf{y}_{i}$ to the fixed effects and additive genetic effects, respectively. The additive genetic effects for all traits were assumed normally distributed with a mean of 0 , a genetic variance of $\sigma_{a_{i}}^{2}$ for trait $i$, and a genetic covariance between traits of $\sigma_{a_{1} a_{2}}:\left[\begin{array}{l}\mathbf{a}_{1} \\ \mathbf{a}_{2}\end{array}\right] \sim N\left[\left(\begin{array}{l}0 \\ 0\end{array}\right), \mathbf{A} \otimes\left(\begin{array}{cc}\sigma_{a_{1}}^{2} & \sigma_{a_{1} a_{2}} \\ \sigma_{a_{1} a_{2}} & \sigma_{a_{2}}^{2}\end{array}\right)\right]$. The residuals were assumed to be normally distributed as well, with a mean of 0 , a residual variance of $\sigma_{e_{i}}^{2}$ for trait $i$, and a residual covariance between traits of $\sigma_{e_{1} e_{2}}:\left[\begin{array}{l}\mathbf{e}_{1} \\ \mathbf{e}_{2}\end{array}\right] \sim N\left[\left(\begin{array}{l}0 \\ 0\end{array}\right), \mathbf{I} \otimes\left(\begin{array}{cc}\sigma_{e_{1}}^{2} & \sigma_{e_{1} e_{2}} \\ \sigma_{e_{1} e_{2}} & \sigma_{e_{2}}^{2}\end{array}\right)\right]$.

Because of long computing times for the bivariate analyses, the data set was randomly split into 5 subsets based on herd. The bivariate analyses were then performed on the 5 subsets and weighted means of the parameters were calculated. See the Appendix for an explanation of the weighting of the parameters resulting from the bivariate analyses of the subsets.

Genetic Correlations with Health, Longevity, Fertility, Metabolic, and Production Traits. Genetic correlations between the resilience indicators and several health, longevity, fertility, metabolic, and production traits were estimated using the multiple trait across country evaluation (MACE) procedure. The MACE procedure is used by Interbull (Interbull, 2017) to evaluate bulls in different countries for the same trait, but it can also be used to estimate genetic correlations between deregressed sire EBV of different traits (Schaeffer, 1994; Klei and Weigel, 1998; Larroque and Ducrocq, 1999). Deregressed EBV are used to make the variance of the EBV independent from their reliabilities and to take out the contributions of the parents (Larroque and Ducrocq, 1999). The MACE procedure was used instead of bivariate analysis because it allows for inclusion of all available records on health, longevity, fertility, metabolic, and production traits in the national population, without the need for the actual data and models that accompany these traits. For the resilience indicators, deregressed sire EBV resulting from the univariate analyses were used as input for the MACE procedure. For the health, longevity, fertility, metabolic, and production traits, deregressed sire EBV from Cooperation CRV and CRV BV from the official run of December 2018 were used as input. All available health indexes and EBV, which were the udder health index (CRV, 2017b), the hoof health index (CRV, 2015), and ketosis resistance in first lactation (Vosman et al., 2015), were included, because resilient cows are expected to be healthier than less-resilient cows. For these 3 traits, a higher value indicates better health or less ketosis. Productive longevity (CRV, 2018c) and the fertility index (CRV, 2017a) were also included, because resilient cows are expected to live longer and to be more fertile than less-resilient cows. For these 2 traits, a higher value indicates better fertility or longevity. In addition, the metabolic traits BCS (CRV, 2018b) and DMI (CRV, 2018e) were included because of the expected relation between resilience and the amount of resources a cow has available to respond to disturbances. For these 2 traits, higher value indicates higher BCS or greater DMI. The production trait fat-protein persistency in first lactation was included to investigate the differences in effect of persistency on RawVar and LnVar. The sire EBV for fat-protein persistency were based on daily EBV for kilograms of fat and protein resulting from a random regression model (CRV, 2018a). For this trait, a higher value indicates a flatter lactation curve. Finally, the production trait milk yield (CRV, 2018a) was included to compare the average daily milk yield calculated in the current AMS data set with the official breeding value for milk yield based on the total Dutch-Flemish cow population.

Genetic Correlations Adjusted for Milk Yield. Considerable genetic correlations between some of the 
Table 2. Variance components $\left(\sigma_{a}^{2}=\right.$ additive genetic variance; $\sigma_{e}^{2}=$ error variance; $\sigma_{p}^{2}=$ phenotypic variance $)$, heritabilities $\left(\mathrm{h}^{2}\right)$, and genetic coefficients of variation (GCV) from the univariate analyses of the resilience indicators (SE in parentheses)



${ }^{1}$ LnVar $=$ variance of deviations from lactation curve, RawVar $=$ variance of raw milk yield, $\mathrm{r}_{\text {auto }}=$ lag-1 autocorrelation of deviations, Skew $=$ skewness of deviations, AMY = average daily milk yield.

${ }^{2} \mathrm{ma}=$ moving average, $\mathrm{mm}=$ moving median, wilm $=$ Wilmink curve, quant $=$ quantile regression.

resilience indicators and average daily milk yield were observed. Therefore, partial genetic correlations between the resilience indicators and the health, longevity, fertility, and metabolic traits, adjusted for average daily milk yield $\left(r_{x y, z}\right)$, were calculated as follows:

$$
r_{x y}, z=\frac{r_{x y}-r_{x z} r_{y z}}{\sqrt{1-r_{x z}^{2}} \sqrt{1-r_{y z}^{2}}}
$$

where $x$ is the resilience indicator, $y$ is the health, longevity, fertility, or metabolic trait, and $z$ is average daily milk yield. The correlations between the resilience indicators and average daily milk yield were genetic correlations obtained from the bivariate analyses. The other correlations were genetic correlations estimated using the MACE procedure.

\section{RESULTS}

\section{Comparison of Resilience Indicators}

The resilience indicators LnVar and RawVar had the highest heritabilities ( 0.20 to 0.24$)$ and genetic coefficients of variation (0.23 to 0.26), whereas Skew had the lowest heritability (0.01 to 0.02$)$ and genetic coefficient of variation (0.05 to 0.10; Table 2). Although RawVar had heritability comparable to LnVar, they were genetically different traits, because the genetic correlations between RawVar and LnVar based on all curve-fitting methods were 0.45 and lower (Table 3). In addition, LnVar, $\mathrm{r}_{\text {auto }}$, and Skew also genetically differed from each other. The genetic correlations between LnVar and $r_{\text {auto }}$ ranged from -0.12 to 0.05 , the genetic correlations between LnVar and Skew ranged from 0.06 to 0.65 , and the genetic correlations between $\mathrm{r}_{\text {auto }}$ and Skew ranged from -0.35 to 0.37 (Table 3). In summary, the genetic correlations show that RawVar, LnVar, $\mathrm{r}_{\text {auto }}$, and Skew are genetically different traits.

\section{Comparison of Lactation Curve-Fitting Methods}

The 4 lactation curve-fitting methods resulted in different means of the resilience indicators (Table 4). We observed the most extreme difference between means for Skew based on a moving median and a Wilmink curve, where the deviations from a Wilmink curve were less skewed than were the deviations from a moving median curve. The genetic variance and heritability also differed between the curve-fitting methods, especially for Skew (Table 2). Skew based on a Wilmink curve genetically differed from the other curve-fitting methods, with genetic correlations ranging from 0.31 to 0.60 (Table 5). The other curve-fitting methods resulted in Skew traits that were genetically more similar, with genetic correlations ranging from 0.81 to 0.95 . In comparison to Skew, LnVar and $r_{\text {auto }}$ had stronger genetic correlations between the curve-fitting methods $(>0.89)$. In summary, for LnVar and $r_{\text {auto }}$ different curve-fitting methods resulted in genetically similar traits, whereas for Skew the different curve-fitting methods resulted in genetically less similar traits.

\section{Genetic Correlations with Health, Longevity, Fertility, Metabolic, and Production Traits}

We found that LnVar and RawVar were the resilience indicators with the strongest genetic correlations 
Table 3. Genetic correlations between the resilience indicators variance, autocorrelation, and skewness for each of the 4 lactation curve-fitting methods, and genetic correlations between variance of deviations from lactation curves and variance of raw milk yield records (SE in parentheses)

\begin{tabular}{|c|c|c|c|c|}
\hline \multirow[b]{2}{*}{ Curve $^{1}$} & \multicolumn{4}{|c|}{ Resilience indicator $^{2}$} \\
\hline & $\begin{array}{l}\text { LnVar } \\
\text { and } \mathrm{r}_{\text {auto }}\end{array}$ & $\begin{array}{l}\text { LnVar } \\
\text { and Skew }\end{array}$ & $\begin{array}{c}\mathrm{r}_{\text {auto }} \\
\text { and Skew }\end{array}$ & $\begin{array}{c}\text { LnVar } \\
\text { and RawVar }\end{array}$ \\
\hline $\mathrm{ma}$ & $-0.12(0.04)$ & $0.51(0.12)$ & $-0.20(0.06)$ & $0.39(0.02)$ \\
\hline $\mathrm{mm}$ & $-0.12(0.03)$ & $0.65(0.10)$ & $-0.35(0.05)$ & $0.37(0.02)$ \\
\hline wilm & $0.05(0.02)$ & $0.06(0.08)$ & $0.37(0.08)$ & $0.45(0.02)$ \\
\hline quant & $-0.04(0.02)$ & $0.49(0.11)$ & $-0.01(0.05)$ & $0.45(0.02)$ \\
\hline
\end{tabular}

${ }^{1} \mathrm{ma}=$ moving average, $\mathrm{mm}=$ moving median, wilm $=$ Wilmink curve, quant $=$ quantile regression.

${ }^{2} \mathrm{LnVar}=$ variance of deviations from lactation curve, $\mathrm{r}_{\text {auto }}=$ lag-1 autocorrelation of deviations, Skew $=$ skewness of deviations, RawVar = variance of raw milk yield.

${ }^{3}$ RawVar is not based on deviations from lactation curves. Therefore, the comparison is between LnVar based on different curves and RawVar.

Table 4. Descriptive statistics of resilience indicators based on different lactation curve-fitting methods, and average daily milk yield

\begin{tabular}{|c|c|c|c|c|c|c|}
\hline Trait $^{1}$ & Curve $^{2}$ & Mean & $\mathrm{SD}$ & Minimum & Maximum & $\begin{array}{l}\text { Number } \\
\text { of cows }\end{array}$ \\
\hline \multirow[t]{4}{*}{ LnVar } & $\mathrm{ma}$ & 0.90 & 0.59 & -1.38 & 3.27 & 198,702 \\
\hline & $\mathrm{mm}$ & 0.87 & 0.61 & -1.59 & 3.33 & 198.712 \\
\hline & wilm & 1.69 & 0.57 & -0.60 & 3.97 & 198,728 \\
\hline & quant & 1.39 & 0.57 & -0.89 & 3.69 & 198,725 \\
\hline RawVar & - & 2.85 & 0.64 & 0.28 & 5.36 & 198,723 \\
\hline \multirow[t]{4}{*}{$r_{\text {auto }}$} & ma & 0.32 & 0.19 & -0.43 & 0.91 & 198,736 \\
\hline & $\mathrm{mm}$ & 0.30 & 0.18 & -0.43 & 0.89 & 198,746 \\
\hline & wilm & 0.65 & 0.18 & -0.10 & 0.98 & 198,343 \\
\hline & quant & 0.56 & 0.19 & -0.22 & 0.98 & 198,580 \\
\hline \multirow[t]{4}{*}{ Skew } & ma & -1.26 & 0.83 & -4.88 & 2.37 & 197,731 \\
\hline & $\mathrm{mm}$ & -1.60 & 1.04 & -6.10 & 2.92 & 197,838 \\
\hline & wilm & -0.82 & 0.65 & -3.48 & 1.85 & 198,452 \\
\hline & quant & -1.27 & 0.74 & -4.38 & 1.87 & 198,197 \\
\hline AMY & - & 26.60 & 4.99 & 6.34 & 46.29 & 198,736 \\
\hline
\end{tabular}

${ }^{1} \mathrm{LnVar}=$ variance of deviations from lactation curve, RawVar $=$ variance of raw milk yield, $\mathrm{r}_{\text {auto }}=$ lag-1 autocorrelation of deviations, Skew $=$ skewness of deviations, AMY $=$ average daily milk yield.

${ }^{2} \mathrm{ma}=$ moving average, $\mathrm{mm}=$ moving median, wilm $=$ Wilmink curve, quant $=$ quantile regression.

Table 5. Genetic correlations between 4 lactation curve-fitting methods for each resilience indicator (SE in parentheses)

\begin{tabular}{lccc}
\hline & & Resilience indicator $^{2}$ \\
\cline { 2 - 4 } Curve $^{1}$ & LnVar & $\mathrm{r}_{\text {auto }}$ & Skew \\
\hline ma and mm & $1.00(0.00)$ & $0.99(0.01)$ & $0.95(0.01)$ \\
ma and wilm & $0.94(0.01)$ & $0.92(0.01)$ & $0.46(0.07)$ \\
ma and quant & $0.98(0.00)$ & $0.97(0.00)$ & $0.81(0.07)$ \\
mm and wilm & $0.94(0.01)$ & $0.90(0.01)$ & $0.31(0.06)$ \\
mm and quant & $0.98(0.00)$ & $0.95(0.01)$ & $0.83(0.06)$ \\
wilm and quant & $0.98(0.00)$ & $0.96(0.01)$ & $0.60(0.03)$ \\
\hline
\end{tabular}

${ }^{1} \mathrm{ma}=$ moving average, $\mathrm{mm}=$ moving median, wilm $=$ Wilmink curve, quant $=$ quantile regression.

${ }^{2} \mathrm{LnVar}=$ variance, $\mathrm{r}_{\text {auto }}=$ autocorrelation, Skew $=$ skewness. 
with the health, longevity, fertility, metabolic, and production traits (Table 6). Both a lower LnVar and a lower RawVar were genetically related to better udder health, better hoof health, better longevity, better fertility, higher BCS, less ketosis, and lower milk yield level. However, LnVar and RawVar differed in the sign (positive or negative) of their genetic correlations with persistency and DMI: LnVar had a positive genetic correlation of only 0.08 to 0.14 with persistency, whereas RawVar had a negative genetic correlation with persistency of -0.51 . With DMI, LnVar had a positive genetic correlation of 0.24 to 0.29 , whereas Raw Var had a negative genetic correlation with DMI of only -0.04 . If we now turn to $r_{\text {auto }}$, we see that its genetic correlations with the health, longevity, fertility, metabolic, and production traits were generally in the same direction as for LnVar: a lower $r_{\text {auto }}$ was genetically related to better health, fertility, and longevity, higher DMI and BCS, and lower milk yield level. However, $r_{\text {auto }}$ had weaker genetic correlations $(-0.21$ to 0.20$)$ with the health, longevity, fertility, metabolic, and production traits than did LnVar. For Skew, the genetic correlations with the health, longevity, fertility, and metabolic traits were, in general, negligible or weak $(-0.25$ to 0.17$)$. A higher Skew was weakly genetically associated with more ketosis, lower BCS, and lower longevity (Table 6 ). The genetic correlations between Skew and average daily milk yield were moderate, and were negative for Skew based on a Wilmink curve $(-0.40)$ but positive for Skew based on the other curve-fitting methods (0.19 to 0.30 ).

Average daily milk yield had a strong genetic correlation (0.95) with official milk yield produced in 305 $\mathrm{d}$ in first lactation (Table 6). This strong genetic correlation indicates that the studied data set is a good representation of the total Dutch-Flemish population. The genetic correlations between LnVar and average daily milk yield were positive and strong (0.75 to 0.79 ), and for both LnVar and average daily milk yield the genetic correlations with the health traits and fertility, BCS, DMI, and ketosis resistance were in the same direction. Interestingly, though, LnVar and average daily milk yield had opposite but weak genetic correlations with productive longevity.

To disentangle the effects of milk yield level and variance in milk yield on the genetic correlations between LnVar and the health, longevity, fertility, and metabolic traits, the original genetic correlations were converted to partial genetic correlations, adjusted for milk yield level. Most partial genetic correlations between LnVar and the health, longevity, fertility, and metabolic traits were weaker (Table 7) than the original genetic correlations had been (Table 6). However, the partial genetic correlations between LnVar and longevity were




Table 7. Partial genetic correlations between resilience indicators based on different lactation curve-fitting methods and health, longevity, fertility, and metabolic traits, estimated using the multiple trait across country evaluation (MACE) procedure and adjusted for average daily milk yield

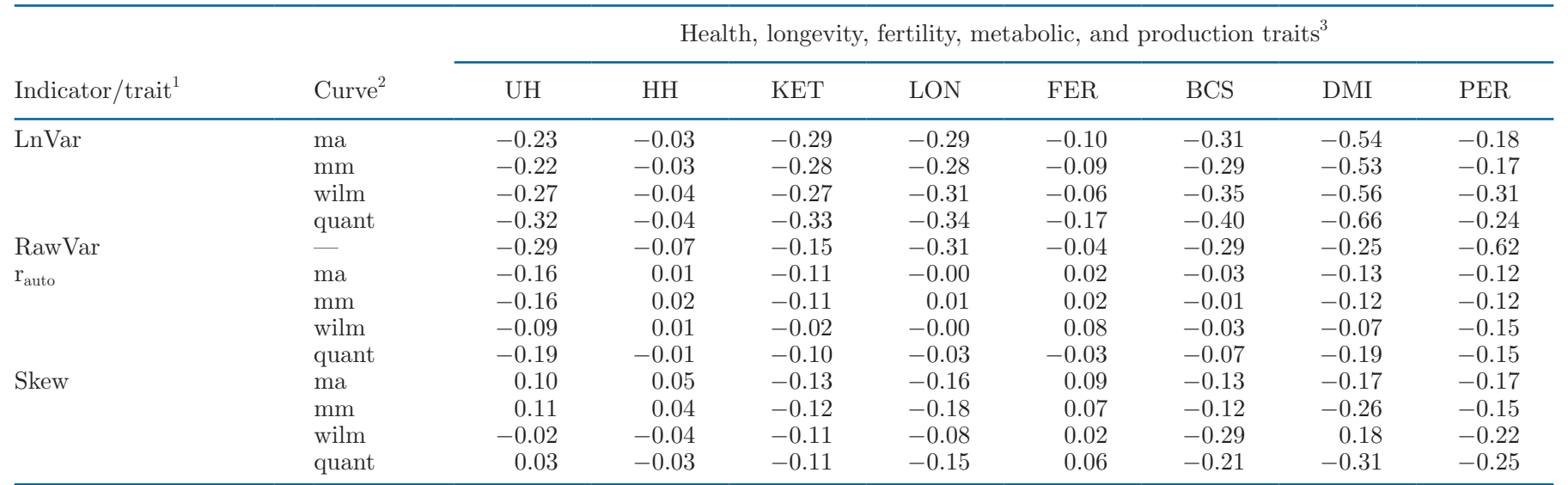

${ }^{1} \operatorname{LnVar}=$ variance of deviations from lactation curve, RawVar $=$ variance of raw milk yield, $\mathrm{r}_{\text {auto }}=$ lag-1 autocorrelation of deviations, Skew $=$ skewness of deviations.

${ }^{2} \mathrm{ma}=$ moving average, $\mathrm{mm}=$ moving median, wilm $=$ Wilmink curve, quant $=$ quantile regression.

${ }^{3} \mathrm{UH}=$ udder health, $\mathrm{HH}=$ hoof health, KET $=$ ketosis resistance in first lactation, $\mathrm{LON}=$ productive longevity, FER $=$ fertility, $\mathrm{PER}=$ persistency in first lactation.

stronger $(-0.28$ to -0.34$)$ than the original genetic correlations. Moreover, the partial genetic correlations between LnVar and DMI were negative $(-0.53$ to -0.66$)$, whereas the original genetic correlations were positive: at an equal milk yield across cows, less variable milk was genetically related to higher DMI. Skew and $r_{\text {auto }}$ had weaker genetic correlations with average daily milk yield than did LnVar (Table 6). The weak genetic correlations with milk yield led to only small differences between the original and the partial genetic correlations. However, the partial genetic correlations of $r_{\text {auto }}$ and Skew with DMI were stronger and, in most cases, had a different sign than did their original genetic correlations with DMI (Table 7). In summary, of the 3 resilience indicators, LnVar had the strongest genetic correlations with the health, longevity, fertility, and metabolic traits. At an equal level of milk yield across cows, lower LnVar was genetically related to better health, longevity, and fertility, and to higher BCS and DMI.

\section{DISCUSSION}

We explored the use of LnVar, $\mathrm{r}_{\text {auto }}$, and Skew of deviations in daily milk yield from different types of lactation curves as indicators of resilience that can be used for breeding. LnVar had the highest heritability and the strongest genetic correlations with health, longevity, fertility, metabolic, and production traits. In addition, the 3 potential resilience indicators were genetically different from each other, whereas the different lactation curve-fitting methods resulted in genetically similar traits for LnVar and $r_{\text {auto }}$ and, to a lesser extent, Skew.

As far as we know, we are the first to perform a genetic analysis on autocorrelation and skewness of a production trait in dairy cattle. However, a similar analysis has been performed on deviations in BW in layer chickens (Berghof et al., 2019a). Although the heritability of autocorrelation based on BW deviations in chickens was similar to the heritability of autocorrelation based on milk yield deviations in cattle, the rest of the results differed between the 2 studies. In chickens, the heritability of skewness was approximately 10 times higher and heritability of variance was approximately 2 times lower than in cattle. In addition, the genetic correlations among the 3 resilience indicators differed widely between the 2 species. The main reason for the differences between the traits in the 2 studies is likely that we used deviations from individually fitted lactation curves, whereas deviations from cohort means were used in the chicken study. Therefore, the interpretation of autocorrelation and skewness differs between the studies. In addition, in this study we had many more records available per animal than in the chicken study.

Whereas autocorrelation and skewness of milk yield have not been studied genetically before, variance has. Compared with previous studies on variance in milk yield, our study provided 2 novelties. The first novelty was that we analyzed the variance in deviations from a lactation curve instead of the variance of raw milk yield values (Elgersma et al., 2018). As expected, fitting a lactation curve removed the effect of persistency on 
variance: LnVar had only a weak positive genetic correlation with persistency (0.08 to 0.14 ), whereas RawVar had a considerable negative genetic correlation with persistency $(-0.51)$. In addition, the genetic correlation between RawVar and LnVar was only moderate. Because of the removed effect of persistency, LnVar has improved value as a resilience indicator compared with variance in raw milk yield, as suggested by Elgersma et al. (2018). The second novelty of this study compared with most previous studies (Rönnegård et al., 2013; Vandenplas et al., 2013; Ehsaninia et al., 2019), but in line with Elgersma et al. (2018), was that we used daily recorded milk yield instead of test-day records to calculate the variance of deviations in milk yield. As expected, the higher number of records per cow used in our study than in the previous studies resulted in a lower environmental variance and a higher heritability of LnVar (Berghof et al., 2019b). Rönnegård et al. (2013), Vandenplas et al. (2013), and Ehsaninia et al. (2019) analyzed their milk yield records with a double hierarchical generalized linear model, which effectively means that they did a genetic analysis immediately on test day milk yield records. This genetic analysis gave them genetic parameters for the mean milk yield and the variance of the deviations from an estimated lactation curve simultaneously. However, we used a 2-step approach and first estimated individual lactation curves and then summarized the deviations into 1 resilience indicator per cow, on which we performed a genetic analysis. In theory, the double hierarchical generalized linear model could be applied to our daily milk yield records as well, but we would expect it to yield EBV and genetic variance in residual variance similar to those of our LnVar (Berghof et al., 2019a), whereas it is computationally much more challenging to apply and more difficult to understand.

Our research focused on fluctuations in milk yield level for defining resilience indicators. However, fluctuations in milk components may also be related to resilience. For example, fluctuations in fat content may indicate resilience to ketosis or rumen acidosis, and fluctuations in SCS may indicate resilience to mastitis (de Haas et al., 2008; Urioste et al., 2012). Rönnegård et al. (2013) and Vandenplas et al. (2013) showed genetic variation in variability of SCS measured on test-days, and Vandenplas et al. (2013) also showed genetic variation in variability of different types of fatty acids. Furthermore, Ehsaninia et al. (2019) showed genetic variation in variability of fat and protein content measured on test-days. It would be interesting to also analyze variability of these traits using daily records and to investigate their genetic correlations with health traits. However, until now daily measurements of milk components have not been available on a large scale.
In our study, as well as in those of Rönnegård et al. (2013), Vandenplas et al. (2013), Elgersma et al. (2018), and Ehsaninia et al. (2019), the variance of (deviations in) milk yield was studied as a resilience indicator. Alternative measures could be the mean of absolute deviations or using the variance only of negative deviations. The mean of absolute deviations may give information similar to that derived from the variance, but a variance gives more weight to large deviations than to small deviations, because a variance is essentially the mean of squared deviations. Because small deviations can be due merely to noise, we expect that the variance is more powerful as a resilience indicator than the mean of absolute deviations. The use of only negative deviations to calculate the variance may seem better than also using positive deviations because disturbances cause drops in milk yield rather than peaks. However, work on daily feed intake in turkeys has shown that variance based only on negative deviations had considerably lower heritability than did the variance based on all deviations, although the genetic correlation between them was 0.98 (H. A. Mulder, unpublished data). Thus, we expect the variance of all deviations to be more informative about resilience than the mean of absolute deviations or the variance of only negative deviations.

All our resilience indicators were based on data from complete lactations up to DIM 350. However, resilience may differ between lactation stages because of differences in disease susceptibility (Ingvartsen et al., 2003). Therefore, it would be interesting to test whether resilience indicators based on data from different lactation stages are genetically different from each other and whether they differ in their genetic correlations with health, longevity, fertility, metabolic, and production traits.

\section{Suitability of Traits Used as Resilience Indicators}

The suitability of a resilience indicator depends on its heritability and its genetic correlations with health, longevity, fertility, metabolic, and production traits. High heritability indicates that family members are more alike than other animals and therefore suggests that the indicator is not simply random noise. Furthermore, resilience is expected to be genetically correlated with better health, longevity, and fertility (Elgersma et al., 2018; Berghof et al., 2019b), higher DMI, higher BCS, and lower milk yield level. The expected correlations with DMI, BCS, and milk yield level can be explained by resource allocation theory. If the demand for resources for milk yield is high, fewer resources are left for coping with disturbances than when demand for resources for milk yield is low. Therefore, a high milk yield can make a cow less resilient. Alternatively, 
a high DMI or BCS can provide a cow with enough resources to cope with disturbances, even at a high milk yield, which makes her more resilient (Rauw, 2008). If a lower LnVar and $\mathrm{r}_{\text {auto }}$ indicate a better resilience, we would expect them to have negative genetic correlations with udder health, hoof health, longevity, fertility, ketosis resistance, DMI, and BCS, and positive correlations with milk yield level. If a higher (more positive) Skew indicates better resilience, we expect it to have positive genetic correlations with udder health, hoof health, longevity, fertility, ketosis resistance, DMI, and BCS, and negative correlations with milk yield level. In the following sections we will discuss which of the resilience indicators behaves most according to these expectations.

Variance. In our results, LnVar based on all curvefitting methods had considerable heritability. In addition, a lower LnVar was genetically correlated with better health, longevity, and fertility, and higher BCS, which was as expected. These results support the hypothesis that LnVar indicates resilience. Most of the genetic correlations with the health, longevity, fertility, and metabolic traits were only moderate, but this is favorable: LnVar should indicate general resilience and not resilience to specific diseases (Putz et al., 2019). Other studies that investigated resilience indicators also found moderate genetic correlations with health and functional traits (Elgersma et al., 2018; Putz et al., 2019). More importantly, all genetic correlations consistently showed that low LnVar was related to a good health and functionality.

Although the health, longevity, fertility, and metabolic traits did not have strong genetic correlations with LnVar, the average daily milk yield did have strong positive correlations. We find 2 possible explanations for these strong genetic correlations. The first is that cows with a higher milk yield level have poorer resilience than do cows with a lower milk yield level. High-producing cows may be expected to have fewer resources available to respond to disturbances than low-producing cows, due to the high resource demand for their milk yield, and thus have a greater tendency to take resources away from production (Rauw, 2008; Berghof et al., 2019b). The second explanation for the strong genetic correlation between LnVar and average daily milk yield is a scale effect (Falconer and Mackay, 1996; Berghof et al., 2019b). A scale effect means that the same disturbance results in a larger drop in milk yield in high-producing cows than in low-producing cows, whereas the size of the drop relative to the production level is equal for cows with different production levels.

Because of the strong positive genetic correlations between LnVar and average daily milk yield, and the generally unfavorable genetic correlation between milk yield and functionality, one could argue that the genetic correlations between LnVar and the health, longevity, fertility, and metabolic traits were mainly caused by milk yield level and not by variability in milk yield. However, the partial genetic correlations between LnVar and most functional traits, adjusted for milk yield, were still considerable and were in the expected direction (low LnVar was related to good health and functionality). These considerable partial genetic correlations indicate that LnVar does contain information about health and functionality that is not covered by milk yield level. Moreover, the partial genetic correlations between LnVar and both DMI and longevity were closer to our expectations of LnVar as a resilience indicator than the original genetic correlations were. The negative partial genetic correlation between LnVar and longevity was stronger than the original one, which was closer to our expectation, because we expected lower LnVar to be related to higher longevity (resilient cows live longer). The change in strength of the genetic correlation between LnVar and longevity after adjustment for milk yield level is caused by the weak genetic correlation between average daily milk yield and longevity: the genetic correlation between LnVar and longevity was "suppressed" by the weak genetic correlation between average daily milk yield and longevity. The partial genetic correlation between LnVar and DMI was quite strong and negative, which means that at an equal level of milk yield across all cows, cows with a low variance in milk yield tend to have higher DMI than do cows with a high variance, which probably gives the lowvariance cows the resources they need to respond to disturbances. The partial genetic correlation between LnVar and DMI was opposite from the original positive genetic correlation that indicated that a higher LnVar was related to a higher DMI. This difference is caused by the positive genetic correlation between milk yield level and DMI: cows with genetically higher milk yields tend to eat more than less-productive cows, but they also have a higher variance in milk yield. The negative partial genetic correlations between LnVar and DMI, and the negative partial genetic correlations between LnVar and longevity, confirm that LnVar corrected for milk yield is informative about resilience.

Although most genetic correlations between LnVar and the functional traits became only slightly weaker after adjusting for milk yield level, the genetic correlations between LnVar and hoof health became negligible. Apparently, LnVar does not contain information about hoof health that is not covered by milk yield level. The negligible partial correlation is probably caused by the inability of the curve-fitting methods to capture longterm declines in milk yield that are typical for lameness 
(Green et al., 2002). Based on the curve-fitting methods applied in this study, LnVar is therefore not suitable for long-term disturbances such as claw disorders.

In summary, LnVar contains information about health and functionality that is not covered by milk yield level. Therefore, LnVar is a promising resilience indicator and seems a good candidate to include in breeding goals. The economic value of resilience is already partly accounted for in breeding goals by health traits. However, resilience has additional economic value, because resilient cows require less time from farmers for checking and monitoring than do less-resilient cows. In addition, resilience may account for the costs of diseases that are not yet included in the breeding goal (Berghof et al., 2019b), such as ketosis and rumen acidosis in the Netherlands (CRV, 2018d). Additional research is needed to determine the correct economic value of LnVar in the breeding goal. Alternatively, a desired-gains approach could be used (Brascamp, 1984). Such an approach should aim for a decrease in LnVar while simultaneously increasing milk yield level, health, longevity, and fertility, and decreasing DMI.

Autocorrelation. The heritability of $\mathrm{r}_{\text {auto }}$ was not high but was still considerable, which suggests that $\mathrm{r}_{\text {auto }}$ contains information and is not just random noise. We expected that the information that $r_{\text {auto }}$ contained would be mostly about the recovery aspect of resilience (Berghof et al., 2019b). A higher $\mathrm{r}_{\text {auto }}$ was expected to indicate longer stretches of negative (or positive) deviations and thus a slower recovery. Because the EBV for the health traits are more informative about resistance to diseases than about recovery from diseases, the genetic correlations between $r_{\text {auto }}$ and the disease traits may be of limited value for validating $r_{\text {auto }}$ as an indicator of recovery time. Indeed, the genetic correlations between $r_{\text {auto }}$ and udder health and hoof health were weak and negligible, respectively. Nevertheless, they were in the expected direction, with a lower $r_{\text {auto }}$ indicating better health. The association between $r_{\text {auto }}$ and udder health could be caused by the positive genetic association between mastitis resistance and recovery rate (Welderufael et al., 2018). Based on this observation, one may expect a positive genetic correlation between $\mathrm{r}_{\text {auto }}$ and LnVar, but we found a weak and negative genetic correlation. In summary, we cannot conclude from our results whether $r_{\text {auto }}$ is informative about recovery time, and more research is needed. If $\mathrm{r}_{\text {auto }}$ turns out to contain information about recovery time that LnVar lacks, the 2 traits could be combined in a resilience index. However, as a single indicator of overall resilience, $\mathrm{r}_{\text {auto }}$ is less suitable than LnVar.

Skewness. Skew contained less genetic variation than LnVar and $r_{\text {auto }}$, indicated by the low GCV, and more noise, indicated by the low heritability. In addition, Skew had weak or unexpected genetic correlations with both LnVar and the health, longevity, fertility, and metabolic traits. For instance, a higher (closer to 0) Skew was expected to indicate better resilience, but was weakly genetically related to shorter longevity, lower BCS, more ketosis, and higher LnVar. Therefore, Skew is not considered a good resilience indicator. The reason that Skew was unable to reflect resilience could be that this trait was too sensitive to incorrect milk yield records: a single outlier could have a large effect on Skew. We were not able to remove all outliers from the data, because to do so would be too strict: we would also remove extreme records that were informative about resilience. Because of its sensitivity to outliers, Skew is not suitable as a resilience indicator using commercial data.

\section{Which Curve-Fitting Method Is Best?}

Because of its heritability and genetic correlations with health, longevity, fertility, metabolic, and production traits, LnVar is the most promising resilience indicator. However, we should also decide which curvefitting method generates the best LnVar. All 4 curvefitting methods resulted in genetically similar LnVar traits (Table 5), which indicates that for LnVar it is not important which curve-fitting method is used. However, we observed small differences in the genetic parameters. Moving average and moving median resulted in the highest heritability, whereas polynomial quantile regression generated the strongest genetic correlations with the health, longevity, fertility, and metabolic traits. To decide which method is best, we can calculate the indirect response in a health trait, such as udder health, that results from selection on reduced LnVar based on the 4 curve-fitting methods. If we consider genomic selection using 20,000 cows in the reference population and we assume that the number of independent chromosomal segments is 1,200 , then the accuracy of a genomic EBV (Daetwyler et al., 2010) for LnVar would be approximately 0.90 based on moving average and moving median, and approximately 0.88 based on polynomial quantile regression and a Wilmink curve. Note that this comparison is not affected by the assumption of the number of independent chromosomal segments; getting an appropriate value is an unresolved scientific issue (Brard and Ricard, 2015). Assuming the selection intensity to be 1 , the genetic improvement in udder health would be $0.24,0.23,0.28$, and 0.25 genetic SD when selection is on LnVar based on moving average, moving median, polynomial quantile regression, and Wilmink curve, respectively. This shows that poly- 
nomial quantile regression would lead to the highest genetic improvement in udder health if selection is on LnVar. The same was observed for hoof health, ketosis, longevity, and fertility. Therefore, polynomial quantile regression is the best curve-fitting method among the methods studied here.

\section{CONCLUSIONS}

All potential resilience indicators explored in this study were heritable, although the heritabilities ranged from low to moderate. We found that LnVar had the highest heritability and the strongest genetic correlations with health, longevity, fertility, and metabolic traits, in the expected direction. Therefore, it has the most potential as an indicator of resilience. The genetic correlations of $r_{\text {auto }}$ with the health, longevity, fertility, and metabolic traits were weak, but more research is needed to investigate whether $r_{\text {auto }}$ could indicate recovery time. Skew had negligible heritability and unexpected genetic correlations with the health, longevity, fertility, and metabolic traits. Skew is thus not considered a good indicator of resilience. The lactation curve-fitting methods on which LnVar was based resulted in genetically similar traits, but selection on LnVar based on quantile regression would consistently result in the largest correlated responses in the health, longevity, and fertility traits. Therefore, we recommend this curve-fitting method. This research is an important stepping stone to further explore the use of logtransformed variance of deviations in milk yield as an indicator that can be used to breed more resilient cows.

\section{ACKNOWLEDGMENTS}

We acknowledge the Dutch Ministry of Economic Affairs (The Hague, the Netherlands; TKI Agri and Food project 16022) and the Breed4Food partners Cobb Europe (Boxmeer, the Netherlands), CRV (Arnhem, the Netherlands), Hendrix Genetics (Boxmeer, the Netherlands), and Topigs Norsvin (Beuningen, the Netherlands) for their financial support. In addition, we acknowledge Cooperation CRV and CRV BV (Arnhem, the Netherlands) for providing the data. Furthermore, we acknowledge European Union's Horizon 2020 Research and Innovation program GenTORE, under grant agreement No. 727213, for financial support. In addition, we acknowledge Claudia Kamphuis (Wageningen Livestock Research, Wageningen, the Netherlands) and Henk Hogeveen (Wageningen University, Wageningen, the Netherlands) and Gerben de Jong (Cooperation CRV, Arnhem, the Netherlands) for their intellectual input and Erik Mullaart (CRV BV, Arnhem, the Netherlands) for his organizational support.

\section{REFERENCES}

Aho, A. V., B. W. Kernighan, and P. J. Weinberger. 1988. The AWK Programming Language. Addison-Wesley Publishing Company, Reading, MA.

Berghof, T. V. L., H. Bovenhuis, and H. A. Mulder. 2019a. Body weight deviations as indicator for resilience in layer chickens. Front. Genet. https://doi.org/10.3389/fgene.2019.01216.

Berghof, T. V. L., M. Poppe, and H. A. Mulder. 2019b. Opportunities to improve resilience in animal breeding programs. Front. Genet. 9:692. https://doi.org/10.3389/fgene.2018.00692.

Brard, S., and A. Ricard. 2015. Is the use of formulae a reliable way to predict the accuracy of genomic selection? J. Anim. Breed. Genet. 132:207-217. https://doi.org/10.1111/jbg.12123.

Brascamp, P. 1984. Selection indices with constraints. Animal Breeding Abstracts 52:645-654.

Brockwell, P. J., and R. A. Davis. 2016. Introduction to Time Series and Forecasting. 3rd ed. R. DeVeaux, S. Fienberg, and I. Olkin, ed. Springer International Publishing, Cham, Switzerland. https:/ /doi.org/10.1007/978-3-319-29854-2.

Colditz, I. G., and B. C. Hine. 2016. Resilience in farm animals: Biology, management, breeding and implications for animal welfare. Anim. Prod. Sci. 56:1961-1983. https://doi.org/10.1071/AN15297.

CRV. 2015. E-30 Claw health index. Accessed Dec. 14, 2018. https:/ /www.cooperatie-crv.nl/wp-content/uploads/2018/10/E_30-Claw -Health-Index-apr2017-_en.pdf.

CRV. 2017a. E-17 Breeding value fertility. Accessed Dec. 14, 2018. https://www.cooperatie-crv.nl/wp-content/uploads/2018/10/E 17-vru_aug2018_en.pdf.

CRV. 2017b. E-27 Breeding value udder health. Accessed Dec. 14, 2018. https://www.cooperatie-crv.nl/wp-content/uploads/2018/ 10/E_27-Udder-Health-April-2017_en.pdf.

CRV. 2018a. E-7 Breeding value estimation of milk production traits with test-day model. Accessed Dec. 14, 2018. https://www .cooperatie-crv.nl/wp-content/uploads/2018/10/E_07-tdm-apr -2018_EN.pdf.

CRV. 2018b. E-8 Breeding value estimation for conformation traits. Accessed Dec. 14, 2018. https://www.cooperatie-crv.nl/wp -content/uploads/2018/10/E_08_eng_augustus-2018.pdf.

CRV. 2018c. E-19 Breeding value for longevity (LON). Accessed Dec. 14, 2018. https://www.cooperatie-crv.nl/wp-content/uploads/ 2018/10/E_19-Longevity_apr2018_eng.pdf.

CRV. 2018d. E-20 NVI. Accessed Sep. 12, 2019. https:/ / www.cooperatie -crv.nl/wp-content/uploads/2018/10/E_20-NVI_aug2018_en.pdf.

CRV. 2018e. E-40 Breeding value dry matter intake. Accessed Dec. 14, 2018. https://www.cooperatie-crv.nl/wp-content/uploads/2018/ 12/E_40-Feed-intake-dec-2018-1.pdf.

Daetwyler, H. D., R. Pong-Wong, B. Villanueva, and J. A. Woolliams. 2010. The impact of genetic architecture on genome-wide evaluation methods. Genetics 185:1021-1031. https://doi.org/10.1534/ genetics.110.116855.

de Haas, Y., W. Ouweltjes, J. ten Napel, J. J. Windig, and G. de Jong. 2008. Alternative somatic cell count traits as mastitis indicators for genetic selection. J. Dairy Sci. 91:2501-2511. https://doi.org/ $10.3168 / \mathrm{jds} .2007-0459$.

Ehsaninia, J., N. Ghavi Hossein-Zadeh, and A. A. Shadparvar. 2019. Estimation of genetic variation for macro- and micro-environmental sensitivities of milk yield and composition in Holstein cows using double hierarchical generalized linear models. J. Dairy Res. 86:145-153. https://doi.org/10.1017/S0022029919000293.

Elgersma, G. G., G. de Jong, R. van der Linde, and H. A. Mulder. 2018. Fluctuations in milk yield are heritable and can be used as a resilience indicator to breed healthy cows. J. Dairy Sci. 101:12401250. https://doi.org/10.3168/jds.2017-13270.

Falconer, D. S., and T. F. C. Mackay. 1996. Introduction to Quantitative Genetics. 4th ed. Pearson Education Limited, Essex, UK.

Friggens, N. C., C. Duvaux-Ponter, M. P. Etienne, T. Mary-Huard, and P. Schmidely. 2016. Characterizing individual differences in animal responses to a nutritional challenge: Toward improved robustness measures. J. Dairy Sci. 99:2704-2718. https://doi.org/10 $.3168 /$ jds.2015-10162. 
Gilmour, A. R., B. J. Gogel, B. R. Cullis, S. J. Welham, and R. Thompson. 2015. ASReml user guide release 4.1 functional specification. VSN International Ltd., Hemel Hempstead, UK.

Green, L. E., V. J. Hedges, Y. H. Schukken, R. W. Blowey, and A. J. Packington. 2002. The impact of clinical lameness on the milk yield of dairy cows. J. Dairy Sci. 85:2250-2256. https://doi.org/10 .3168/jds.S0022-0302(02)74304-X.

Hill, W. G., and H. A. Mulder. 2010. Genetic analysis of environmental variation. Genet. Res. (Camb.) 92:381-395. https://doi.org/10 $.1017 /$ S0016672310000546.

Ingvartsen, K. L., R. J. Dewhurst, and N. C. Friggens. 2003. On the relationship between lactational performance and health: Is it yield or metabolic imbalance that cause production diseases in dairy cattle? A position paper. Livest. Prod. Sci. 83:277-308. https:// doi.org/10.1016/S0301-6226(03)00110-6.

Interbull. 2017. Interbull code of practice-Methods of international evaluation. Accessed Dec. 14, 2018. http://www.interbull.org/ib/ cop chap5.

Klei, L., and K. A. Weigel. 1998. A method to estimate correlations among traits in different countries using data on all bulls. Interbull Bull. 17:8-14. https://journal.interbull.org/index.php/ib/article/ download/486/483.

Koenker, R. 2005. Quantile regression. Econometric Society Monographs. A. Prat and S. Bonhomme, ed. Cambridge University Press, New York, NY. https://doi.org/10.1017/CBO9780511754098.

Koenker, R. 2018. quantreg: Quantile Regression. R package version 5.36. https://CRAN.R-project.org/package=quantreg.

Larroque, H., and V. Ducrocq. 1999. An indirect approach for the estimation of genetic correlations between longevity and other traits. Interbull Bull. 21:128-136. https://journal.interbull.org/index .php/ib/article/download/454/452.

Mulder, H. A., P. Bijma, and W. G. Hill. 2007. Prediction of breeding values and selection responses with genetic heterogeneity of environmental variance. Genetics 175:1895-1910. https://doi.org/ 10.1534/genetics.106.063743.

Pool, M. H., and T. H. E. Meuwissen. 2000. Reduction of the number of parameters needed for a polynomial random regression test day model. Livest. Prod. Sci. 64:133-145. https://doi.org/10.1016/ S0301-6226(99)00166-9.

Putz, A. M., J. C. S. Harding, M. K. Dyck, F. Fortin, G. S. Plastow, J. C. M. Dekkers, and PigGen Canada. 2019. Novel resilience phenotypes using feed intake data from a natural disease challenge model in wean-to-finish pigs. Front. Genet. 9:660. https://doi.org/ 10.3389/fgene.2018.00660

Rauw, W. M. 2008. Resource Allocation Theory Applied to Farm Animal Production. CABI Publishing, Wallingford, UK.

Revilla, M., N. C. Friggens, L. P. Broudiscou, G. Lemonnier, F. Blanc, L. Ravon, M. J. Mercat, Y. Billon, C. Rogel-Gaillard, N. Le Floch, J. Estellé, and R. Muñoz-Tamayo. 2019. Towards the quantitative characterisation of piglets' robustness to weaning: A modelling approach. Animal 13:2536-2546. https://doi.org/10.1017/ S1751731119000843.

Rönnegård, L., M. Felleki, W. F. Fikse, H. A. Mulder, and E. Strandberg. 2013. Variance component and breeding value estimation for genetic heterogeneity of residual variance in Swedish Holstein dairy cattle. J. Dairy Sci. 96:2627-2636. https://doi.org/10.3168/ jds.2012-6198.

SanCristobal-Gaudy, M., J. M. Elsen, L. Bodin, and C. Chevalet. 1998. Prediction of the response to a selection for canalisation of a continuous trait in animal breeding. Genet. Sel. Evol. 30:423-451. https://doi.org/10.1186/1297-9686-30-5-423.

Schaeffer, L. R. 1994. Multiple-country comparison of dairy sires. J. Dairy Sci. 77:2671-2678. https://doi.org/10.3168/jds.S0022 -0302(94)77209-X.
Schaeffer, L. R., J. Jamrozik, G. J. Kistemaker, and B. J. Van Doormaal. 2000. Experience with a test-day model. J. Dairy Sci. 83:1135-1144. https://doi.org/10.3168/jds.S0022-0302(00)74979 -4 .

Scheffer, M. 2009. Critical Transitions in Nature and Society. Princeton University Press, Princeton, NJ.

Scheffer, M., J. E. Bolhuis, D. Borsboom, T. G. Buchman, S. M. W. Gijzel, D. Goulson, J. E. Kammenga, B. Kemp, I. A. van de Leemput, S. Levin, C. M. Martin, R. J. F. Melis, E. H. van Nes, L. M. Romero, and M. G. M. Olde Rikkert. 2018. Quantifying resilience of humans and other animals. Proc. Natl. Acad. Sci. USA 115:11883-11890. https://doi.org/10.1073/pnas.1810630115.

Sell-Kubiak, E., P. Bijma, E. F. Knol, and H. A. Mulder. 2015. Comparison of methods to study uniformity of traits: Application to birth weight in pigs. J. Anim. Sci. 93:900-911. https://doi.org/10 $.2527 /$ jas.2014-8313.

Swalve, H. H. 2000. Theoretical basis and computational methods for different test-day genetic evaluation methods. J. Dairy Sci. 83:1115-1124. https://doi.org/10.3168/jds.S0022-0302(00)74977 -0 .

Urioste, J. I., J. Franzén, J. J. Windig, and E. Strandberg. 2012. Genetic relationships among mastitis and alternative somatic cell count traits in the first 3 lactations of Swedish Holsteins. J. Dairy Sci. 95:3428-3434. https://doi.org/10.3168/jds.2011-4739.

van der Werf, J. H., M. E. Goddard, and K. Meyer. 1998. The use of covariance functions and random regressions for genetic evaluation of milk production based on test day records. J. Dairy Sci. 81:3300-3308. https://doi.org/10.3168/jds.S0022-0302(98)75895 $-3$.

Vandenplas, J., C. Bastin, N. Gengler, and H. A. Mulder. 2013. Genetic variance in micro-environmental sensitivity for milk and milk quality in Walloon Holstein cattle. J. Dairy Sci. 96:5977-5990. https://doi.org/10.3168/jds.2012-6521.

Veerkamp, R. F., C. L. Gerritsen, E. P. Koenen, A. Hamoen, and G. de Jong. 2002. Evaluation of classifiers that score linear type traits and body condition score using common sires. J. Dairy Sci. 85:976-983. https://doi.org/10.3168/jds.S0022-0302(02)74157-X.

Vosman, J., G. De Jong, H. Eding, and H. Knijn. 2015. Genetic evaluation for ketosis in the Netherlands based on FTIR measurements Interbull Bull. 49:1-5. https://journal.interbull.org/index.php/ib/ article/view/1365/1433.

Welderufael, B. G., P. Løvendahl, D. J. de Koning, L. L. G. Janss, and W. F. Fikse. 2018. Genome-wide association study for susceptibility to and recoverability from mastitis in Danish Holstein cows Front. Genet. 9:141. https://doi.org/10.3389/fgene.2018.00141.

Wilmink, J. B. M. 1987. Adjustment of test-day milk, fat and protein yield for age, season and stage of lactation. Livest. Prod. Sci. 16:335-348. https://doi.org/10.1016/0301-6226(87)90003-0.

Zeileis, A., and G. Grothendieck. 2005. zoo: S3 Infrastructure for regular and irregular time series. J. Stat. Softw. 14:1-27. https://doi .org/10.18637/jss.v014.i06.

\section{ORCIDS}

M. Poppe (ㄴ) https://orcid.org/0000-0001-7801-3942

R. F. Veerkamp ๑ https://orcid.org/0000-0002-5240-6534

M. L. van Pelt (1) https://orcid.org/0000-0003-1315-1329

H. A. Mulder (ㅇ https://orcid.org/0000-0003-2124-4787 


\section{APPENDIX}

\section{Bivariate Analyses Using Subsets}

Because of the long computing time, genetic correlations between the resilience indicators were estimated on subsets of the complete data. Weighted averages were subsequently estimated, which are presented in this paper. Here we provide additional information about the aggregation of the genetic correlations estimated on subsets of the data.

The complete data set was randomly split into 5 subsets based on herd. On each subset, genetic correlations between resilience indicators were estimated. Weighted averages of the genetic correlations $(\bar{x})$ were estimated as follows:

$$
\bar{x}=\frac{\sum_{i=1}^{5} w_{i} x_{i}}{\sum_{i=1}^{5} w_{i}},
$$

where $w_{i}$ was the number of animals in subset $i$ and $x_{i}$ was the estimated genetic correlation of subset $i$. Weighted standard deviations (SD) of the genetic correlations $(s)$ were estimated thus:

$$
s=\sqrt{\frac{\sum_{i=1}^{5} w_{i}\left(x_{i}-\bar{x}\right)^{2}}{\frac{4 \sum_{i=1}^{5} w_{i}}{5}}} .
$$

To calculate the standard error of the weighted average genetic correlations, the weighted SD were divided by the square root of 5 . In addition to the weighted genetic correlations, weighted residual and phenotypic correlations were also calculated, as shown in Appendix Table A1. 
Poppe et al.: MILK FLUCTUATIONS AS A RESILIENCE INDICATOR

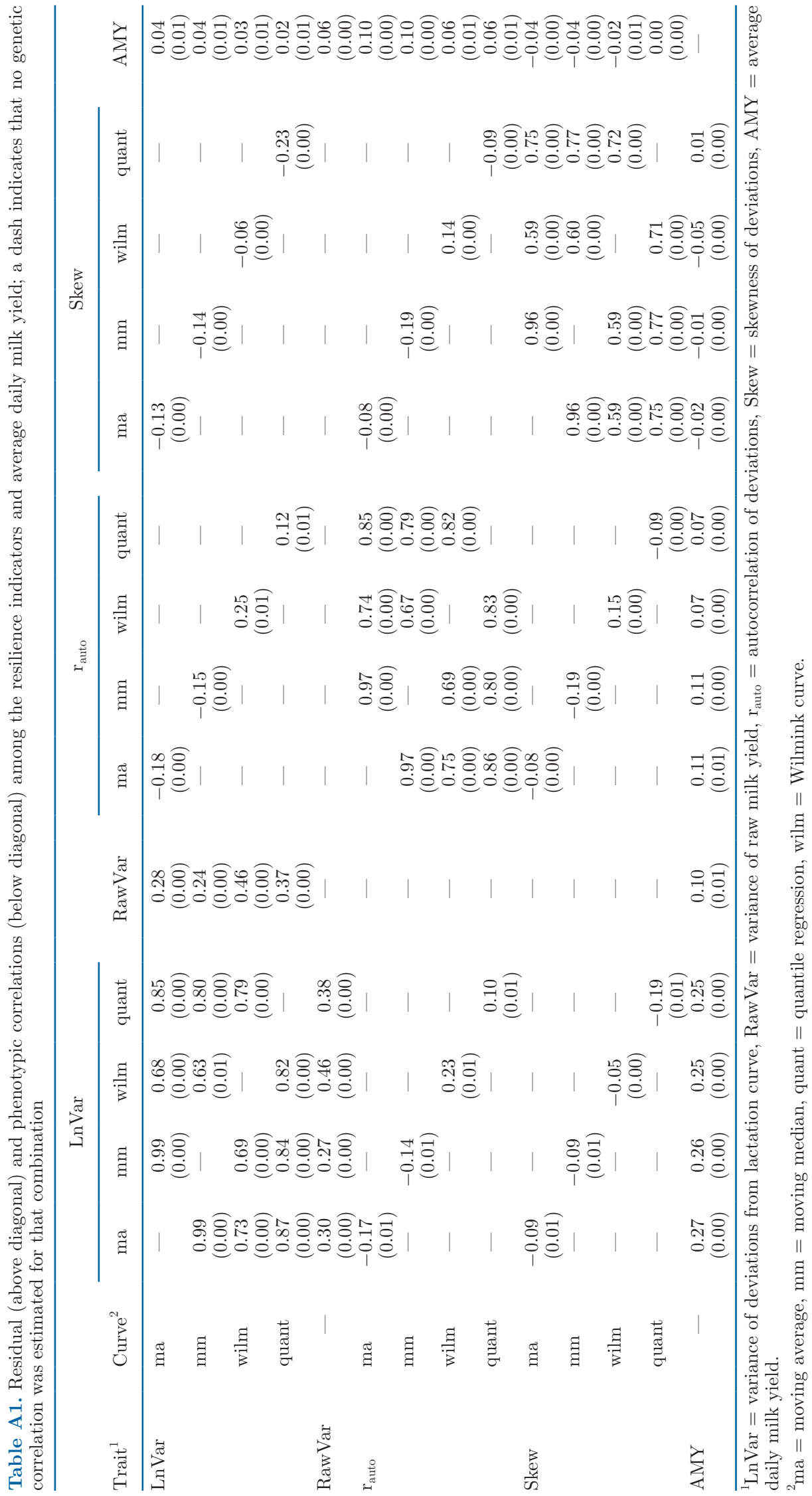

